\title{
Prevalence and characteristics of dual sensory impairment (hearing and vision) in a veteran population
}

\author{
Sherri L. Smith, PhD; ${ }^{*}$ Loren W. Bennett, OD, MPH; Richard H. Wilson, PhD \\ James H. Quillen Department of Veterans Affairs Medical Center, Mountain Home, TN; East Tennessee State Univer- \\ sity, Johnson City, TN
}

\begin{abstract}
With the increasing aging population, the number of veterans presenting with dual sensory impairment (DSI) (vision and hearing impairments) will increase. This study determined the prevalence and clinical characteristics of DSI in a veteran population receiving healthcare from the Department of Veterans Affairs (VA). A retrospective review was conducted on 400 charts randomly selected from a database of 1,472 unique veterans enrolled in the audiology and optometry outpatient clinics during a 1-year period. Depending on definition criteria, hearing impairment prevalence was $41.6 \%$ to $74.6 \%$, vision impairment/blindness prevalence was $7.4 \%$, and DSI prevalence was $5.0 \%$ to $7.4 \%$. The vision impairment/ blindness prevalence governed the DSI prevalence. By age, DSI prevalence ranged from $0 \%$ (among veterans $<65$ years) to $>20 \%$ (among veterans $85+$ years). The complexities encountered in defining DSI are discussed and suggestions are made for determining standardized definitions. More research is needed to determine the unique characteristics of individuals with DSI and their impact on VA resources.
\end{abstract}

Key words: auditory impairment, dual sensory impairment, hearing impairment, hearing loss, legal blindness, prevalence, rehabilitation, veterans, vision impairment, vision loss.

\section{INTRODUCTION}

Hearing and vision impairments are common chronic conditions in older adults that have been studied extensively as single sensory disabilities. Dual sensory impairment (DSI), or concurrent hearing and vision impairments, also occurs in older adults but is less well understood. Historically, rehabilitation of hearing and vision impairments has been accomplished separately by audiologists and optometrists/ophthalmologists, respectively, with little interaction between the disciplines for individuals with DSI. Clinicians in these disciplines are aware of the importance of auditory and vision function for activities of daily living and communication; however, joint rehabilitation programs for individuals with DSI are few. For the audiologist, individuals with vision impairment pose unique challenges in the audiological rehabilitative process [1]. Often hearing impairment is treated via small amplification devices such as hearing aids, auditory perception training, communication strategies training, and counseling. Individuals with hearing impairment often rely on vision cues to improve communication (viz.,

\footnotetext{
Abbreviations: $\mathrm{CI}=$ confidence interval; $\mathrm{dB} \mathrm{HL}=\mathrm{dB}$ hearing level; DSI = dual sensory impairment; HFPTA = high-frequency pure tone average; ICD-9-CM = International Classification of Diseases-9th Revision, Clinical Modification; PTA = pure tone average; RR\&D = Rehabilitation Research and Development; VA = Department of Veterans Affairs; VHA = Veterans Health Administration, $\mathrm{WHO}=$ World Health Organization.

*Address all correspondence to Sherri $L$. Smith, PhD; VA Medical Center, Audiology-126, Mountain Home, TN 37684; 423-926-1171, ext 7569; fax: 423-979-3403.

Email: sherri.smith@va.gov

DOI: 10.1682/JRRD.2007.02.0023
} 
speech reading) and to use and care for their amplification devices (e.g., changing batteries, daily cleaning). Likewise, individuals with hearing impairment may produce unique challenges in low-vision and blind rehabilitation programs. Individuals with vision impairment and legal blindness often are provided with optical and nonoptical low-vision devices, which may require auditory cues to assist with ambulation or "talking" devices such as watches, books on tape, or bar-coded label readers to increase independence. Effective rehabilitation of individuals with DSI, therefore, may be hindered by the inability of the individual to use alternate sensory input in a compensatory fashion. Rehabilitation efforts also might be enhanced if more were known about the functional and psychosocial impact of DSI as compared with those of a single sensory impairment.

Although the evidence is not conclusive, some literature suggests that DSI is more debilitating than a single sensory impairment, whereas other literature suggests that the vision impairment component of DSI is the sensory impairment that has the main impact on the individual. Studies suggest that individuals with DSI have an increased risk for reduced function with activities of daily living (e.g., ambulation, eating) and instrumental activities of daily living (e.g., telephone use, medication management) [2-5] compared with individuals with a single sensory impairment, whereas other studies suggest that hearing impairment does not further reduce functioning beyond the vision impairment alone [6-7]. Older adults with DSI also are more likely than individuals with single sensory impairments to exhibit depressive symptoms [8-9]. One study reported that hearing impairment did not increase depressive symptoms in participants with vision impairment [10]. In comparison with a single sensory impairment, DSI is associated with lower healthrelated quality of life [11] and increased risk of mortality [12]. Interestingly, one report found that individuals with vision impairment also may have poorer hearing sensitivity than individuals without vision impairment, suggesting an underlying biological contribution to impairments with both senses [11]. A better understanding of the impact that DSI has on individuals is necessary to assist the rehabilitative process for older veterans.

Older age is a well-established risk factor for sensory impairment, and as the U.S. population ages, DSI will become more prevalent. Older adults represent the fastest growing segment of the U.S. population, especially the very old (i.e., 85+ years) [13]. Current estimates predict that between 2002 and 2020, the 85+ veteran population will increase by 58 percent and the $90+$ veteran population will increase by 235 percent [14]. Inevitably, DSI will have a growing impact on future health care trends in the Veterans Health Administration (VHA).

As a result of the foreseeable growing population of older veterans, VHA is increasingly interested in DSI, as evidenced by the recent conference hosted by the Department of Veterans Affairs (VA) Rehabilitation Research and Development (RR\&D) Center of Excellence National Center for Rehabilitative Auditory Research [15]. The consensus from this conference was that scientific evidence about DSI is lacking in many areas, including but not limited to diagnostics, rehabilitation, psychosocial implications, and cognitive functioning. Interest in DSI also is evidenced by the recent addition of a DSI diagnosis code, V49.85, effective October 1, 2007, to the International Classification of Diseases-9th Revision, Clinical Modification (ICD-9-CM) manual. Although this diagnosis code is generic in that DSI is defined as any degree of combined hearing impairment and vision impairment, the addition of this code to the ICD-9-CM manual demonstrates that DSI is a newly recognized problem (see http://www.cdc.gov and http:// www.cms.hhs.gov).

DSI can be modeled schematically as a two-channel continuum with an auditory channel and a vision channel. In this DSI model, which is illustrated in Figure 1, channel one is the auditory channel (top) and channel two is the vision channel (bottom). Normal auditory and visual function is at the left extreme on the continuum, whereas the complete loss of auditory and visual function (i.e., deaf and blind) is at the right extreme. The area between

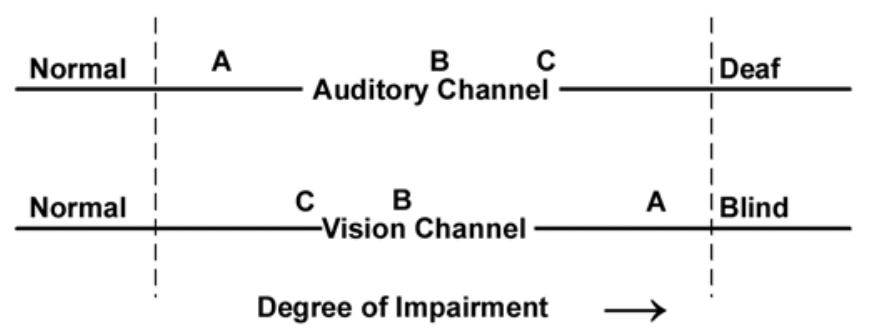

Figure 1.

Schematic of dual sensory impairment model is shown along with degree of auditory and vision impairments of three individuals (A, B, and $\mathrm{C}$ ). Person $\mathrm{A}$ has mild hearing impairment and severe vision impairment, person B has moderate impairments in both senses, and person $\mathrm{C}$ has severe hearing impairment and mild vision impairment. 
the dashed vertical lines represents a segment of the continuum that includes individuals who retain some degree of sensory function with both senses or individuals who potentially have DSI, which is the most likely case of veterans seeking services from VHA. The main issue is that the functional and psychosocial impacts of DSI created by the combination of various degrees of single sensory impairment are unknown. Several reasons contribute to this inconclusive evidence. One reason is that standardized definitions of the hearing and vision impairment components are not used to determine DSI. A second reason is that the various combinations of the two sensory impairments may affect individuals differently.

The three cases of individuals with DSI (A, B, and C in Figure 1) illustrate the complexities of combining both hearing impairment and vision impairment in a single individual. Person A has a mild hearing impairment combined with a severe vision impairment, person B has moderate impairments in both senses, and person $C$ has a severe hearing impairment and a mild vision impairment. The problems with defining DSI are exacerbated by the difficulties involved in integrating the single sensory impairments into a unified definition of DSI. For example, the functional and psychosocial impacts of a moderate impairment in each sense in person $B$ are unknown but may be considered less than additive, additive, or in some instances, compounded. For person B, therefore, the degree of DSI may be moderate or worse, but functionally, the combined effect of the sensory impairments is unknown. The development of a standardized DSI definition is fundamental to a full understanding of the impact of DSI.

Variability in DSI prevalence data reflects the lack of a standard definition. Data from several studies indicate that the prevalence rate of DSI ranges from 1.3 to 35.0 percent in adult populations [2-3,8-12,16-17]. Table 1 summarizes the hearing and vision impairment definitions and the overall DSI prevalence rate from the just-mentioned studies. The top portion of the table summarizes prevalence rates obtained from studies that used self-report techniques, which are Likert-type response scales, to define hearing and vision impairments. In these studies, the overall prevalence rate of DSI ranged from 1.3 to 21.0 percent. The middle portion of the table summarizes two studies that used objective standard clinical results to classify the two sensory impairments. Finally, the bottom portion of the table summarizes two studies that combined objective and subjective clinical results to classify the impairments. In these final two studies, the vision impairment was classified based on standard clinical results, whereas the hearing impairment was defined based on substandard clinical measures or self-reported data. For example, Lupsakko et al. defined hearing impairment based on whether or not the interviewer had difficulty communicating with the participant, whether or not the participant reported hearing difficulty as a main health problem or had a hearing aid [8]. The DSI prevalence rates of these latter two studies that used subjective/ objective-based definitions differed by 6.0 percent. Overall, the large range of prevalence rates fundamentally is attributable to the following: (1) the different definitions used to classify DSI; (2) the different age groups studied; and (3) the different populations studied, such as population- or institutional-based samples.

In the prevalence studies summarized in Table 1, the degree of each single sensory impairment used to establish the DSI definitions also varied. For example, Lupsakko et al. defined vision impairment as $<20 / 60$ near or distance best-corrected binocular visual acuity [8], whereas Chia et al. defined vision impairment as $<20 / 40$ in the better eye [11]. Some definitions of hearing impairment were based on thresholds obtained without hearing aids being worn [11], whereas other definitions were based on thresholds obtained with hearing aids being worn when available [17]. Regardless of the definition used, that the studies summarized in Table 1 had similar overall prevalences (i.e., in the $6 \%-13 \%$ range) is interesting. The three exceptions to this were Brennan et al. [2], Lieberman et al. [17], and Lam et al. [12]. First, Brennan et al. reported a 21 percent DSI prevalence in a large sample from a community-based population $(N=$ 5,151) [2], which is probably high because of the selfreport data used to classify DSI. Second, Lieberman et al. reported a 35 percent prevalence rate in an institutionalized population [17], consistent with other studies showing higher single sensory impairment rates in this unique group of older adults. Third, Lam et al. found a low DSI prevalence rate of 1.3 percent by including a wider community-based age range that included adults as young as 18 [12], which probably skewed the data.

The different prevalence rates and the mixed evidence associated with the impact of DSI demonstrate that DSI is multifaceted and complex. Another complication is that the population with DSI is largely older, adding further heterogeneity. The complexity associated with defining and understanding DSI, however, is determined 
JRRD, Volume 45, Number 4, 2008

Table 1.

Hearing and vision impairment definitions, overall dual sensory impairment prevalence rates, and ages studied from several published studies.

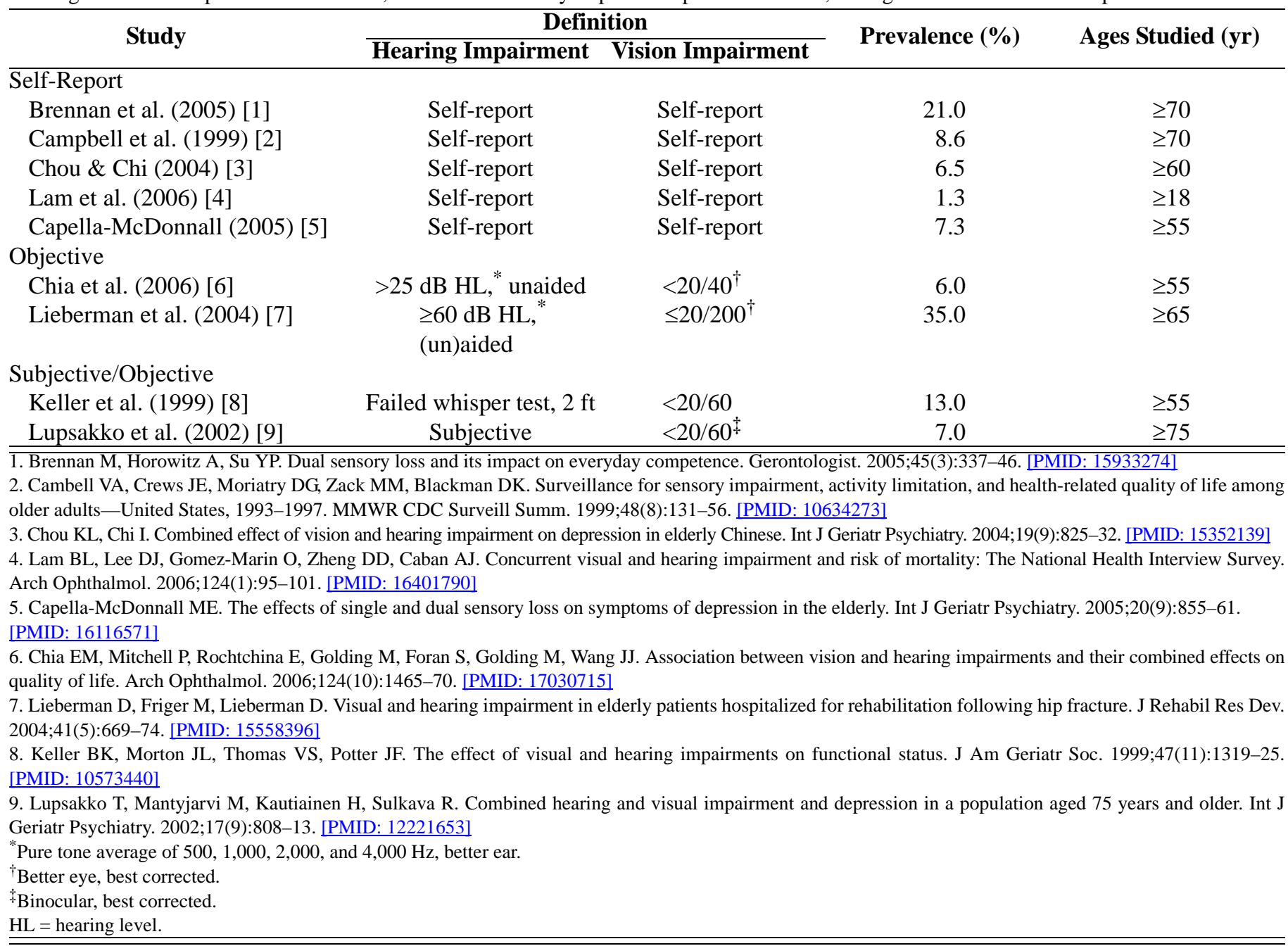

primarily by the definitions used to define the respective single sensory impairments.

For years, hearing impairment typically has been defined by the unaided pure tone average (PTA) of the air-conduction thresholds at 500, 1,000, and 2,000 $\mathrm{Hz}$. Historically, this three-frequency PTA has been recognized as highly correlated with the auditory threshold for speech stimuli [18-19]. Perhaps because of this relation between pure tone and speech thresholds, the threefrequency PTA has become the gold standard by which hearing impairment is described. Within VHA, the degree of hearing impairment for each ear is described with the three-frequency PTA [20]. The PTAs used to describe the degree of hearing impairment are defined as follows: (1) normal, <25 dB hearing level (dB HL) [20]; (2) mild, 25 to $40 \mathrm{~dB} \mathrm{HL}$; (3) moderate, 41 to $55 \mathrm{~dB}$ HL;
(4) moderate-severe, 56 to $70 \mathrm{~dB}$ HL; (5) severe, 71 to $90 \mathrm{~dB}$ HL; and (6) profound, >90 dB HL. Although these PTAs have limitations in characterizing hearing impairment, we used this metric in the initial analysis of the study data as a starting point for the definition of hearing impairment. Subsequently, we investigated a second PTA weighted more with high-frequency information as an alternative pure tone metric. This high-frequency PTA (HFPTA) included the thresholds at 1,000, 2,000, and $4,000 \mathrm{~Hz}$ and has been shown to have a stronger relationship to the understanding of speech signals presented in background noise than traditional PTA [21-23].

Vision impairment is defined as a functional limitation of the eyes (e.g., reduced visual acuity, visual field loss, contrast sensitivity reduction, glare disability) that cannot be corrected with standard glasses or contact 
lenses. The definitions and classification of levels of vision impairment and blindness vary. For example, the World Health Organization (WHO) defines blindness as a profound vision impairment with best-corrected visual acuity worse than 20/400, low vision (or moderate-severe vision impairment) as visual acuity between 20/70 and $20 / 400$, and normal to near-normal vision as $20 / 60$ or better in the better eye [24]. The definition of legal blindness used in this country requires a best-corrected distance visual acuity of 20/200 or worse in the better eye or a visual field reduction to $20^{\circ}$ in diameter or less. This standard measurement determines, for example, eligibility for rehabilitative programs (such as VHA Blind Rehabilitation Center) or Social Security disability benefits [25-26]. In another departure from WHO terminology, many large population-based studies use visually impaired to refer to a best-corrected visual acuity worse than 20/40 in the better eye [27-28]. Most states also use $20 / 40$ visual acuity as an unrestricted driver's license requirement [29]. Needless to say, a multitude of definitions confound our understanding of DSI.

The present study estimated the prevalence of DSI in a population of veterans receiving healthcare from the VA and described their clinical hearing and visual characteristics. To accomplish this goal, we formulated an operational definition based on standardized definitions of the single sensory impairments and on available objective data. Although the goal of the present study was not to develop a standard definition of DSI via an interactive process, the operational definition used represents a starting place in the process of estimating the prevalence of DSI in a veteran sample. The final goal of this project was to generate a discussion about DSI within VHA so that appropriate preparations could be made to identify and better manage the foreseeable growing veteran population affected by DSI [30].

\section{METHODS}

\section{Operational Definition of Dual Sensory Impairment}

To estimate the prevalence of DSI in our veteran sample, we formulated an operational definition of DSI by using previously described categories of each sensory impairment. The visual impairment component of DSI was defined as best corrected visual acuity of worse than 20/40 in the better eye, which included the U.S. definition of legal blindness (i.e., best corrected visual acuity of $\leq 20 / 200$ in the better eye, or visual field $<20^{\circ}$ ). Vision impairment is categorized based on the better eye, whereas hearing impairment is defined for each ear. For consistency with the vision definitions and for practical reasons, the hearing impairment component of DSI is defined only for the ear with the better pure tone thresholds. The hearing impairment definitions are based on unaided thresholds (i.e., thresholds obtained without a hearing aid); therefore, a moderate hearing impairment or worse in the better ear (i.e., >40 dB HL PTA) was chosen, because individuals with this degree of hearing impairment would likely have considerable listening difficulties in most situations without the use of amplification devices.

\section{Procedures}

A retrospective chart review was completed to obtain data on veterans who sought treatment in both the optometry and audiology outpatient clinics at the VA medical center in Mountain Home, Tennessee, between June 1, 2004, and May 31, 2005. Because patients seen in these two clinics had standardized clinical data on their vision and hearing status in their medical charts, a query from a computerized hospital database generated a list of all unique patients seen in these two clinics $(N=1,472)$. Owing to the relationship between the prevalence of DSI and age, the patient list was stratified by age into the following four groups: $(1)<65$ years ( $n=505$, range $=44-64$ years), (2) 65 to 74 years $(n=363)$, (3) 75 to 84 years $(n=485)$, and (4) $85+$ years $(n=119$, range $=85-95$ years). We randomly selected 100 patients from each of the four age groups.

The following data were obtained from the medical charts to determine the prevalence and clinical characteristic of veterans with DSI: (1) age at the time of the visit, (2) best-corrected distance visual acuity in the better eye, (3) etiology of the vision impairment, (4) pure tone, airconduction thresholds from 250 to $8,000 \mathrm{~Hz}$ in the better ear, (5) word-recognition performance in quiet in the better ear, and (6) hearing aid possession. This study was approved by the local institutional review board and the local research and development committee before its initiation.

Audiologists and optometrists collected the clinical data obtained from the medical charts. All audiometric testing (Grason-Stadler audiometer, Model 61, Cardinal Health; Dublin, Ohio) was conducted in a double-walled sound-treated booth (IAC; Bronx, New York) by using insert earphones (EAR-3As; Indianapolis, Indiana) [31]. 
The pure tone thresholds were measured with use of the modified Hughson-Westlake procedure [32]. Word recognition was measured with the Northwestern University Auditory Test No. 6 as recorded on the VA compact disc [33]. Typically, word recognition was evaluated at two levels $20 \mathrm{~dB}$ apart, with the lower level minimally at 60 $\mathrm{dB}$ HL. Distance visual acuity was measured with a projected Snellen chart with the best spectacle correction in place. When available, visual field results contributing to the definition of legal blindness were reviewed from either the Humphrey Automated Visual Field Analyzer (Model HFA II-i, Carl Zeiss; Dublin, California) or the manual Goldmann perimeter (Haag-Streit International; Mason, Ohio).

\section{Statistical Analyses}

We calculated the prevalence data to estimate the proportion of veterans with hearing impairment alone, with vision impairment alone, and with DSI for each age group. The prevalence rate is a single value calculated from a sample that provides the best estimation of the population value [34]. For prevalence rates that were not too small (e.g., $\geq 5 \%$ ), the $95 \%$ confidence interval (CI) was calculated. This interval provides a range of values in which the population proportion of veterans with the sensory impairments in each age group falls with 95 percent certainty. For small prevalence rates (e.g., $<5 \%$ ) with sample sizes of 100 , predetermined $95 \%$ CIs based on a binomial distribution were used [35]. A test of significance of difference in the prevalence across the age groups was performed with an omibus chi-square test [36].

The overall prevalence collapsed across age groups, termed $P_{\text {overall }}$, of the sensory impairments and of DSI also was calculated. Owing to the stratified sample, the overall prevalence was calculated by

$$
P_{\text {overall }}=\sum p_{i} w_{i}
$$

in which $p_{i}$ is the prevalence rate in the $i$ age group and $w_{i}$ is the proportion of veterans in the $i$ age group estimated by the following proportions of veterans in each of the four age groups in the original database $(N=1,472)$ : (1) 34.3 percent, <65 years; (2) 24.7 percent, 65 to 74 years; (3) 32.9 percent, 75 to 84 years; and (4) 8.1 percent, $85+$ years. The $95 \% \mathrm{CI}$ estimate for the overall prevalence was determined by

$$
P_{\text {overall }} \pm(1.96) \sqrt{\frac{\sum \frac{w_{i}^{2} p_{i}\left(1-p_{i}\right)}{n_{i}}}{n_{i}}},
$$

in which $n_{i}$ is the sample size in the $i$ age group [37]. SPSS 14.0 for Windows (SPSS Inc; Chicago, Illinois) was used to conduct all other statistical analyses.

\section{RESULTS}

Because the operational definition of DSI was derived from a combination of the definitions of the single sensory impairments, the prevalences and 95\% CIs of hearing impairment, vision impairment/legal blindness, and DSI were calculated (Equations 1-2). Additionally, the prevalences and CIs for the four age groups were determined for each sensory impairment and for DSI.

\section{Hearing Impairment}

The mean pure tone, air-conduction thresholds in the better ear for each age group are listed in Table 2, along with the mean values for the traditional PTA and the word-recognition score for the better ear. As expected with this sample of older participants, the mean audiogram and PTA illustrate a high-frequency hearing impairment for each age group that increases with increasing age. Word-recognition performance declines with age.

The degree of hearing impairment was categorized for each age group. Recall that the degree of hearing impairment, which was defined by the PTA of thresholds at $500,1,000$, and $2,000 \mathrm{~Hz}$ in the better ear, is categorized as normal, mild, moderate, moderate-severe, severe, and profound [20]. Table 3 lists the percentage of participants and 95\% CIs for each hearing impairment category by age group. In general, the data in the table indicate that hearing impairment increased significantly with increasing age $\left(\chi^{2}(15, N=400)=161.3, p<0.001\right)$. Only a few older adults had severe $(n=17)$ and profound $(n=3)$ hearing impairments. Overall, based on the weighted formula (Equation 1), 41.6 percent (95\% CI = 37.1-46.1) of the participants had a moderate or worse hearing impairment ( $>40 \mathrm{~dB}$ HL PTA).

Data related to best-corrected hearing thresholds were unavailable; however, the number of participants who possessed hearing aids was available and is listed in Table 4 for each hearing impairment category. Consider 
Table 2.

Mean \pm standard deviation pure tone thresholds, pure tone average (PTA) in decibels hearing level (ANSI, 2004), and word-recognition score (WRS) in percent correct are listed for four age groups.

\begin{tabular}{|c|c|c|c|c|c|c|c|c|c|c|}
\hline \multirow{2}{*}{ Group } & \multirow{2}{*}{ Age (yr) } & \multirow{2}{*}{$N$} & \multicolumn{6}{|c|}{ Frequency $(\mathrm{Hz})$} & \multirow{2}{*}{ PTA } & \multirow{2}{*}{ WRS } \\
\hline & & & 250 & 500 & 1,000 & 2,000 & 4,000 & 8,000 & & \\
\hline $1(<65)$ & & 100 & $22.7 \pm 11.8$ & $24.0 \pm 12.3$ & $24.8 \pm 13.2$ & $37.2 \pm 19.5$ & $59.2 \pm 22.0$ & $54.3 \pm 21.6$ & $28.6 \pm 12.7$ & $86.2 \pm 14.1$ \\
\hline$(65$ & & & $7.3 \pm 13.5$ & $29.6 \pm 14.1$ & $32.0 \pm 16.1$ & $50.9 \pm 21.9$ & $69.6 \pm 16.5$ & $68.7 \pm 17.8$ & 37.5 & $75.9 \pm 21.2$ \\
\hline $4(85-95)$ & $87.6 \pm 2.4$ & 100 & $39.8 \pm 16.7$ & $44.3 \pm 16.1$ & $50.8 \pm 16.1$ & $63.9 \pm 12.8$ & $80.6 \pm 12.9$ & $82.1 \pm 12.0$ & $53.1 \pm 13.2$ & $47.3 \pm 26.3$ \\
\hline
\end{tabular}

Table 3.

Degree of hearing impairment (decibels hearing level; ANSI, 2004) displayed as \% (95\% confidence interval) for participants in four age groups. Hearing impairment category was based on pure tone average at $500,1,000$, and 2,000 $\mathrm{Hz}$ in better ear.

\begin{tabular}{lcccc}
\hline \begin{tabular}{c} 
Hearing Impairment \\
\multicolumn{1}{c}{ Category }
\end{tabular} & $<\mathbf{6 5} \mathbf{~ Y r}$ & $\mathbf{6 5 - 7 4} \mathbf{~ Y r}$ & $\mathbf{7 5 - 8 4} \mathbf{Y r}$ & $\mathbf{8 5 +}$ Yr \\
\hline Normal (<25) & $41(31.4-50.6)$ & $20(12.2-27.8)$ & $3(0.1-8.3)$ & $2(0.2-6.9)$ \\
Mild (25-40) & $46(36.2-55.8)$ & $44(34.3-53.7)$ & $31(21.9-40.1)$ & $18(10.5-25.5)$ \\
Moderate (41-55) & $9(3.4-14.6)$ & $27(18.3-35.7)$ & $34(24.7-43.3)$ & $33(23.8-42.2)$ \\
Moderate-Severe (56-70) & $3(0.06-8.3)$ & $6(1.4-10.7)$ & $21(13.0-29.0)$ & $42(32.3-51.7)$ \\
Severe (71-90) & $1(0.03-5.4)$ & $2(0.2-6.9)$ & $9(3.4-14.6)$ & $5(0.7-9.27)$ \\
Profound (>91) & $0(0.0-3.6)$ & $1(0.03-5.4)$ & $2(0.2-6.9)$ & $0(0.0-3.6)$ \\
\hline
\end{tabular}

first only the data in the two columns associated with the $500,1,000$, and 2,000 $\mathrm{Hz}$ PTA (the data in the two columns associated with the HFPTA will be considered in a subsequent section). The data associated with the traditional PTA show that the majority of the 400 participants had hearing aids ( $n=357,89 \%)$. Interestingly, 40 of the 66 participants with so-called "normal" hearing had hearing aids. This number may seem alarmingly high for individuals with normal hearing; however, recall that hearing impairment was based on the traditional PTA of $500,1,000$, and 2,000 Hz, which emphasizes the low and mid frequencies. Participants defined as having normal hearing could have a PTA $<25 \mathrm{~dB}$ HL but have a highfrequency hearing impairment that warranted hearing aid intervention. To better understand why a high percentage of participants in the study had hearing aids, the mean audiogram for each hearing impairment category is illustrated in Figure 2. Notice that the participants defined as having normal hearing (filled circles) based on the traditional PTA had a moderate hearing impairment at 4,000 and $8,000 \mathrm{~Hz}$, which are above the frequency range used with the PTA. These participants most likely understand conversation well in one-on-one or small group settings in quiet environments but may have difficulty understanding conversations in background noise (e.g., restaurants, parties) or when visual cues are limited (e.g., in the car, on the telephone) [21-22]. These communication difficulties in participants defined as having normal hearing
Table 4.

Number of participants with hearing aids (HAs) in each hearing impairment category collapsed across all age groups. Impairment category based on traditional pure tone average (PTA) $(500,1,000$, $2,000 \mathrm{~Hz})$ and high-frequency PTA (HFPTA) $(1,000,2,000,4,000 \mathrm{~Hz})$ in decibels hearing level.

\begin{tabular}{lrrrrrr}
\hline \multirow{2}{*}{$\begin{array}{l}\text { Hearing Impairment } \\
\text { Category }\end{array}$} & \multicolumn{2}{c}{ PTA } & & \multicolumn{2}{c}{ HFPTA } \\
\cline { 2 - 3 } \cline { 5 - 6 } & $\boldsymbol{n}$ & $\begin{array}{c}\text { No. with } \\
\text { HA }\end{array}$ & & $\boldsymbol{n}$ & $\begin{array}{c}\text { No. with } \\
\text { HA }\end{array}$ \\
\hline Normal & 66 & 40 & & 19 & 8 \\
Mild & 139 & 127 & & 65 & 45 \\
Moderate & 103 & 99 & & 113 & 106 \\
Moderate-Severe & 72 & 71 & & 138 & 135 \\
Severe & 17 & 17 & & 59 & 57 \\
Profound & 3 & 3 & & 6 & 6 \\
\hline \hline
\end{tabular}

would be perceived as substantial enough to warrant hearing aid intervention. Classifying hearing impairment based on the traditional PTA is misleading and beyond the original intended use of the PTA.

Normal hearing sensitivity is depicted by the shaded region in Figure 2 with thresholds $<25 \mathrm{~dB}$ HL at each frequency. The remaining pure tone thresholds in Figure 2 represent the mean hearing sensitivity of the participants for each of the five remaining categories of hearing impairment. The pure tone configuration of hearing impairment is high frequency in nature, which is typical with age-related hearing impairment. The numbers in the 


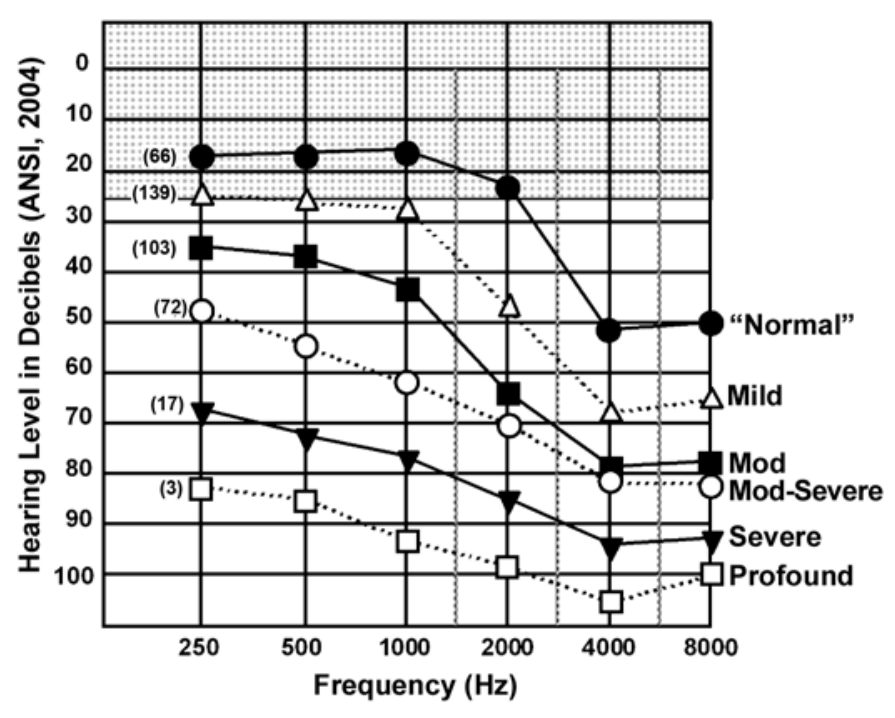

Figure 2.

Mean audiogram of better ear is illustrated for each degree of hearing impairment category, which was based on three-frequency pure tone average at 500, 1,000, and 2,000 Hz. Shaded region depicts normal hearing category across all frequencies. Numbers in parentheses represent number of veterans with that degree of hearing impairment. Mod = Moderate.

parentheses to the left of each function indicate the number of participants who were defined as having the corresponding category of hearing impairment. Based on the traditional PTA, these numbers suggest that the majority of participants had mild or moderate hearing impairments and a few participants had severe or profound hearing impairments.

Because the traditional PTA was misleading in characterizing hearing impairment, we reclassified the pure tone data by using the HFPTA of the thresholds at 1,000, 2,000, and 4,000 Hz. The HFPTA gives more weight to the higher frequencies and, as several studies suggest, is a better indicator of hearing function than the traditional PTA [21-23,38]. Table 5 lists the age, pure tone thresholds, traditional PTA, HFPTA, and word-recognition scores for each hearing impairment category that was based on the traditional PTA (top portion) and on the HFPTA (bottom portion). As age increases, pure tone thresholds increase and word-recognition scores decrease, which are expected relations among these three variables. For each hearing-impairment category, the HFPTA is on average $12 \mathrm{~dB}$ poorer than the traditional PTA. The effect of this $12 \mathrm{~dB}$ shift is reflected in the distribution of participants in the categories of hearing impairment. For example, when we used the traditional PTA, 139 participants were in the mild category; this number decreased to 65 participants when we used the HFPTA. If the HFPTA were used to determine the overall prevalence of hearing impairment, then the prevalence rate of the participants with a moderate hearing impairment or worse would be 74.6 percent $(95 \% \mathrm{CI}=70.3$ 78.9). When we used the HFPTA, 30 percent more participants were categorized with at least a moderate hearing impairment when compared with the prevalence rate of 41.6 percent when we used the traditional PTA.

To understand which pure tone metric better defines functional hearing impairment in terms of hearing aid usage, consider again the data in Table 4, which lists the number of participants in each hearing impairment category who had VA-issued hearing aids. The data in the right-most columns associated with the HFPTA show that only 8 participants with normal hearing based on the HFPTA had hearing aids compared with 40 participants with normal hearing based on the traditional PTA. The data demonstrate that classifying hearing impairment with the HFPTA is more consistent with the functional hearing status as indicated by hearing aid usage than is traditional PTA. From this viewpoint, the HFPTA is a more valid indicator of hearing impairment than is the traditional PTA.

\section{Vision Impairment and Legal Blindness}

The participants were classified into three vision categories based on their best-corrected distance visual acuity in the better eye: (1) normal/near normal, $\geq 20 / 40$; (2) vision impairment, 20/50 to 20/100; or (3) legal blindness, $\leq 20 / 200$. The prevalence rate and $95 \%$ CI of each of the three vision categories for each age group is listed in Table 6. The majority of participants in each age group had corrected visual acuities in the normal/near-normal category. For example, all the participants $<65$ years of age were defined as having normal/near-normal visual acuity. A total of 26 patients were diagnosed with vision impairment across all age groups, and an additional 17 were diagnosed with legal blindness. The overall prevalence of vision impairment and legal blindness was 7.4 percent (95\% CI $=5.0-9.8)$, which is a weighted value (Equation 1). Finally, the data indicate a significant increase in the prevalence of vision impairment and legal blindness with increasing age $\left(\chi^{2}(6, N=400)=42.0, p<\right.$ 0.001). 
Table 5.

Mean \pm standard deviation pure tone thresholds, pure tone average (PTA) (500, 1,000, 2,000 Hz), and high-frequency PTA (HFPTA) (1,000, 2,000, 4,000 Hz) in decibels hearing level, and word-recognition score (WRS) in percent correct are listed for six categories of hearing impairment as determined by traditional PTA and HFPTA.

\begin{tabular}{|c|c|c|c|c|c|c|c|c|c|c|c|}
\hline \multirow{2}{*}{$\begin{array}{c}\text { Hearing } \\
\text { Impairment } \\
\text { Category }\end{array}$} & \multirow{2}{*}{ Age (yr) } & \multirow{2}{*}{$n$} & \multicolumn{6}{|c|}{ Frequency (Hz) } & \multirow{2}{*}{ PTA } & \multirow{2}{*}{ HFPTA } & \multirow{2}{*}{ WRS } \\
\hline & & & 250 & 500 & 1,000 & 2,000 & 4,000 & 8,000 & & & \\
\hline \multicolumn{12}{|l|}{ PTA } \\
\hline Normal & $62.4 \pm 9.0$ & 66 & $17.1 \pm 7.8$ & $15.8 \pm 6.7$ & $15.2 \pm 6.1$ & $22.7 \pm 11.0$ & $52.3 \pm 20.1$ & $51.0 \pm 20.5$ & $17.9 \pm 4.4$ & $30.1 \pm 9.2$ & $92.7 \pm 9.5$ \\
\hline Mild & $70.2 \pm 10.6$ & 139 & $23.9 \pm 9.8$ & $25.9 \pm 8.1$ & $27.2 \pm 6.9$ & $46.5 \pm 13.3$ & $66.6 \pm 15.4$ & $64.7 \pm 17.6$ & $33.2 \pm 4.5$ & $46.8 \pm 8.1$ & $79.4 \pm 16.3$ \\
\hline Moderate & $77.7 \pm 9.2$ & 103 & $33.8 \pm 11.1$ & $37.1 \pm 9.3$ & $43.8 \pm 8.8$ & $63.9 \pm 10.8$ & $78.5 \pm 12.3$ & $78.0 \pm 13.0$ & $48.3 \pm 4.3$ & $62.1 \pm 6.7$ & $57.4 \pm 23.8$ \\
\hline $\begin{array}{l}\text { Moderate- } \\
\text { Severe }\end{array}$ & $83.0 \pm 7.8$ & 72 & $47.5 \pm 12.1$ & $55.0 \pm 7.8$ & $61.9 \pm 6.3$ & $70.0 \pm 7.3$ & $81.2 \pm 12.8$ & $82.6 \pm 12.0$ & $62.3 \pm 4.1$ & $71.0 \pm 6.3$ & $43.0 \pm 22.4$ \\
\hline Severe & $80.3 \pm 7.3$ & 17 & $67.6 \pm 17.7$ & $72.9 \pm 14.0$ & $76.8 \pm 11.3$ & $85.6 \pm 9.5$ & $92.9 \pm 12.9$ & $90.0 \pm 5.3$ & $79.2 \pm 6.5$ & $85.1 \pm 6.8$ & $24.6 \pm 21.9$ \\
\hline Profound & $74.7 \pm 8.5$ & 3 & $83.3 \pm 7.6$ & $85.0 \pm 5.0$ & $93.3 \pm 7.6$ & $98.3 \pm 5.8$ & $105.0 \pm 13.2$ & $100.0 \pm 8.7$ & $92.2 \pm 1.0$ & $98.9 \pm 3.5$ & $9.3 \pm 10.1$ \\
\hline \multicolumn{12}{|l|}{ HFPTA } \\
\hline Normal & $57.2 \pm 8.3$ & 19 & $15.3 \pm 6.8$ & $16.1 \pm 7.4$ & $13.4 \pm 5.3$ & $13.9 \pm 6.1$ & $29.5 \pm 9.6$ & $35.8 \pm 14.4$ & $14.5 \pm 5.5$ & $18.9 \pm 3.4$ & $95.2 \pm 9.5$ \\
\hline Mild & $64.9 \pm 9.5$ & 65 & $21.9 \pm 8.7$ & $22.5 \pm 9.5$ & $20.5 \pm 7.3$ & $27.3 \pm 8.0$ & $51.7 \pm 11.8$ & $50.1 \pm 19.2$ & $23.4 \pm 6.0$ & $33.2 \pm 4.6$ & $91.7 \pm 9.5$ \\
\hline Moderate & $71.6 \pm 10.4$ & 113 & $25.0 \pm 12.2$ & $26.4 \pm 11.0$ & $27.7 \pm 9.4$ & $47.2 \pm 11.7$ & $68.7 \pm 9.3$ & $67.8 \pm 12.9$ & $33.8 \pm 6.8$ & $47.9 \pm 4.1$ & $79.4 \pm 15.7$ \\
\hline $\begin{array}{l}\text { Moderate- } \\
\text { Severe }\end{array}$ & $77.8 \pm 9.3$ & 138 & $35.9 \pm 14.4$ & $39.9 \pm 14.1$ & $46.2 \pm 13.2$ & $63.9 \pm 8.7$ & $77.9 \pm 9.6$ & $78.0 \pm 11.9$ & $50.0 \pm 9.0$ & $62.7 \pm 4.3$ & $56.4 \pm 22.0$ \\
\hline Severe & $81.8 \pm 9.5$ & 59 & $47.4 \pm 17.7$ & $53.6 \pm 15.7$ & $62.7 \pm 12.9$ & $77.2 \pm 7.6$ & $92.8 \pm 11.5$ & $88.6 \pm 9.6$ & $64.7 \pm 9.2$ & $77.6 \pm 5.0$ & $35.2 \pm 22.3$ \\
\hline Profound & $79.2 \pm 7.3$ & 6 & $79.2 \pm 9.2$ & $83.3 \pm 6.1$ & $89.2 \pm 8.6$ & $98.3 \pm 7.5$ & $105.0 \pm 8.9$ & $96.7 \pm 6.8$ & $90.3 \pm 2.5$ & $97.5 \pm 3.1$ & $5.6 \pm 8.8$ \\
\hline
\end{tabular}

Table 6.

Prevalence rate displayed as \% (95\% confidence interval) for each vision category and age group.

\begin{tabular}{lcccc}
\hline \multicolumn{1}{c}{ Vision Category } & $<\mathbf{6 5} \mathbf{~ Y r}$ & $\mathbf{6 5 - 7 4} \mathbf{~ Y r}$ & $\mathbf{7 5 - 8 4} \mathbf{Y r}$ & $\mathbf{8 5 +}$ Yr \\
\hline Normal/Near-Normal & $100(96.4-100)$ & $96(92.2-99.8)$ & $87(80.4-93.6)$ & $74(65.4-82.6)$ \\
Vision Impairment & $0(0.0-3.6)$ & $3(0.06-8.3)$ & $7(2.0-12.0)$ & $16(8.8-23.2)$ \\
Legal Blindness & $0(0.0-3.6)$ & $1(0.03-5.4)$ & $6(1.4-10.7)$ & $10(4.1-15.9)$ \\
\hline \hline
\end{tabular}

The diagnoses associated with vision impairment and legal blindness are given in Table 7. Some participants had multiple diagnoses, resulting in excess total numbers and percentages. Macular degeneration was the most common diagnosis, followed by glaucoma and cataracts. These diagnoses are consistent with several populationbased studies that have demonstrated the role of age as a risk factor for vision impairment [27,39].

\section{Dual Sensory Impairment}

For operational purposes, the participants in this study with a moderate or worse hearing impairment and a vision impairment or legal blindness were defined as having DSI. Because the HFPTA was a more appropriate pure tone metric than the traditional PTA, we calculated DSI using the HFPTA as the hearing impairment component in the DSI definition. In the top portion of Table 8, the prevalence rate and $95 \%$ CIs of those participants cat- egorized with DSI are listed for each age group and for the entire sample. The data associated with the HFPTA show a significant increase in the prevalence of DSI with age $\left(\chi^{2}(3, N=400)=41.6, p<0.001\right)$. In the youngest age group, 0 percent of the participants were categorized with DSI, whereas the prevalence in the oldest age group increased to 26.0 percent. The overall DSI prevalence was 7.4 percent (Equation 1).

The DSI prevalence also was calculated by using the traditional PTA, because this metric has been used over the years as the gold standard definition of hearing impairment or hearing function. The data in the bottom portion of Table 8 list the prevalence and 95\% CIs of participants who were categorized as having DSI when we used the traditional PTA. The data show a significant increase in DSI with increasing age, ranging from 0 percent in the $<65$ year age group to 22.0 percent in the $85+$ year age group $\left(\chi^{2}(3, N=400)=42.1, p<0.001\right)$. With 
Table 7.

Prevalence rate displayed as \% (95\% confidence interval) of participants categorized with dual sensory impairment based on high-frequency pure tone average (HFPTA) $(1,000,2,000,4,000 \mathrm{~Hz})$ and pure tone average (PTA) $(500,1,000,2,000 \mathrm{~Hz})$ for each age group ( $n=100 \mathrm{per}$ group) and overall $(N=400)$.

\begin{tabular}{lccrrr}
\hline Method & $<\mathbf{6 5 ~ Y r}$ & $\mathbf{6 5 - 7 4 ~ Y r}$ & $\mathbf{7 5 - 8 4}$ Yr & 85+ Yr & Overall \\
\hline HFPTA & $0(0.0-3.6)$ & $4(0.2-7.8)$ & $13(6.4-19.6)$ & $26(17.4-34.6)$ & $7.4(6.0-8.7)$ \\
PTA & $0(0.0-3.6)$ & $1(0.03-5.4)$ & $9(3.4-14.6)$ & $22(13.7-30.3)$ & $5.0(3.1-6.9)$ \\
\hline \hline
\end{tabular}

Table 8.

Medical diagnoses displayed as total No. (\%) of participants defined as having vision impairment $(n=26)$ and legal blindness $(n=17)$.

\begin{tabular}{lccccc}
\hline \multicolumn{1}{c}{ Category } & Cataracts & $\begin{array}{c}\text { Macular } \\
\text { Degeneration }\end{array}$ & Glaucoma & $\begin{array}{c}\text { Diabetic } \\
\text { Retinopathy }\end{array}$ & Other \\
\hline Vision Impairment & $8(30.8)$ & $12(46.2)$ & $8(30.8)$ & $4(15.4)$ & $6(23.1)$ \\
Legal Blindness & $3(17.6)$ & $14(82.4)$ & $4(23.5)$ & $0(0.0)$ & $0(0.0)$ \\
\hline Note: Percentages exceed 100 because participants had multiple medical etiologies. & & 38 \\
\hline
\end{tabular}

the use of the traditional PTA, the overall prevalence of DSI was 5.0 percent.

\section{DISCUSSION}

This study determined the prevalence and clinical characteristics of DSI in veterans who receive their healthcare from the VA. The overall prevalence of DSI in veterans at the VA medical center in Mountain Home was 5.0 or 7.4 percent, depending on whether the traditional PTA or the HFPTA was used to define hearing impairment. These overall prevalence rates represent the prevalence of DSI in the original database $(N=1,472)$, which was determined based on the weighted formula (Equation 1). Interestingly, all participants $65+$ years of age who had vision impairment or legal blindness also had a moderate or worse hearing impairment when hearing impairment was defined based on the HFPTA, resulting in the same overall prevalence for vision impairment/ blindness and DSI (i.e., 7.4\%). The reason for these same prevalence rates is that the smaller prevalence rate determines the maximum combined prevalence rate; i.e., the smaller prevalence rate is the governor. The organizational ramifications of potentially all older veterans diagnosed with vision impairment or legal blindness also being diagnosed with DSI warrants further investigation.

The DSI prevalence rates observed in this study are based on a sample of veterans who are enrolled in both the audiology and optometry clinics. For two reasons, the 5.0 or 7.4 percent prevalence of DSI can be generalized with some degree of certainty to the veteran population receiving healthcare from the VA. First, the majority of patients with vision impairment/blindness enrolled at the VA for their healthcare participate in the optometry clinic, especially considering the services available through the VA blind rehabilitation program. Second, as the data demonstrated, the prevalence of DSI is limited by the prevalence of vision impairment/blindness. If the DSI prevalence were governed by the hearing component, then the generalization would not be appropriate. For a variety of reasons, including eligibility and selfdenial, many patients with hearing loss who receive their healthcare from the VA are not enrolled in the audiology clinic. Applying the current data to veterans who do not receive their healthcare from the VA is beyond the scope of this project.

A direct comparison of the DSI prevalence rates between the current study and any other study is difficult owing to variability in DSI definitions. The 5.0 or 7.4 percent overall prevalence rate of DSI in the current study falls within the range of prevalence rates reported in the majority of studies (Table 1). This finding was expected because our sample is an outpatient population of older adults, whereas the low rate of 1.3 percent reported by Lam et al. included young adults [12] and the high rate of 35 percent rate reported by Lieberman et al. was found with an institutionalized population of older adults [18].

The different prevalence rates among the various studies demonstrate the complexities involved with defining DSI. A standardized DSI definition is needed before VHA can assess accurately the characteristics of this population and develop policies. Single sensory impairments and their corresponding relationships to disability have been extensively studied, and VHA has 
well-established programs to treat hearing and vision impairments. The psychosocial and functional impacts of DSI are less well understood than those of its single sensory components. Intuitively, DSI seemingly confers a greater risk for disability than a single sensory impairment, but the existing evidence is mixed. Classification of DSI around established criteria would enable a better understanding of the special needs this group might have and facilitate communication among providers to identify and improve healthcare outcomes.

The results of this investigation and other considerations indicate that the operational definition of DSI used in this study needs to be revised by incorporating other measures of sensory function. As indicated in the "Introduction" section, hearing impairment has been defined by the traditional PTA. Although pure tone thresholds provide frequency-specific information about the auditory system, the thresholds are not necessarily predictive of auditory performance in the communication process. Further, the data in Figure $\mathbf{2}$ and Table $\mathbf{4}$ demonstrate that a definition of hearing impairment based on traditional PTA lacks face validity. The HFPTA is a better pure tone metric than the traditional PTA in that it places more emphasis on the higher frequencies, which are important for communication, especially in background noise [2123,38,40-44]. Our recommendations are that the definition of DSI should consider (1) an HFPTA based on the pure tone thresholds at 1,000, 2,000, and 4,000 Hz; (2) auditory measures of speech recognition both in quiet and noise; and (3) an instrument that evaluates the ability of the listener to understand speech based on both acoustic and visual cues both with and without amplification.

Likewise, visual acuity as measured in this study represents best-corrected, high-contrast distance visual function, which only moderately reflects visual performance or disability. Additional testing, such as contrast sensitivity or reading speed, has been used to further define visual function but is not commonly available in clinical practice [45]. Clinical testing could be augmented to include assessments of activities of daily living, instrumental activities of daily living, localization, cognitive function, and psychosocial assessments, among others. Although classifying patients with DSI based on a pure tone metric and visual acuity may be more efficient because the data are readily available, further testing with the aforementioned functional measures of impairment may provide a more ecologically valid definition of DSI. Other considerations for enhancing the single sensory impairment definitions include whether or not to incorporate the better eye or ear (performance-wise) or to incorporate both eyes and ears in the definitions. The use of presenting habitual sensory situation (i.e., uncorrected or with current glasses or hearing aid intervention) should be considered also, because individuals may be effectively impaired by not using any sensory aids or having an outdated optical correction.

With the single sensory impairments defined, the next challenge is how to combine the single sensory impairments to formulate a pragmatic DSI definition. The complexities in developing a DSI definition stem from the fact that the single sensory impairment definitions are difficult to equate and that significant variation within each sensory impairment exists. Recall our DSI model illustrated in Figure 1. The point on each channel of our model that causes the negative impact of DSI to exceed the impact of the single sensory impairment is unknown. Combining the various degrees of hearing and vision impairments into one definition, therefore, is difficult. A more detailed iterative approach might be to develop a classification scheme with various levels of DSI, such as mild, moderate, or severe. Because of the reliance on the other senses when one has a sensory impairment, the individual with DSI must be rehabilitated to optimize both senses. Having a classification scheme that determines the level of DSI may be valuable for developing rehabilitation approaches and policies.

\section{CONCLUSIONS}

Over the next several years, veterans will be aging, with the most significant increases in the oldest age group. This demographic shift also will increase the number of veterans presenting with DSI who need VA healthcare services. The issues encompassing DSI are numerous and poorly understood but of vested interest to VHA [15]. Our insufficient knowledge of DSI is rooted in the lack of a standard classification scheme to define the population. In order to understand better the unique needs of the DSI population and develop appropriate rehabilitation programs, we must first be able to identify individuals with DSI by using an agreed upon standard definition, which currently does not exist. Once a standard definition of DSI is established, then mechanisms can be developed to implement appropriate programs within VHA as needed. 


\section{ACKNOWLEDGMENTS}

We wish to thank Laura Fleenor, Crystal Carnell, and Pamela Key for their assistance with the chart review. We also acknowledge the statistical consultation that Dr. Tiejian Wu provided on this project.

This material was based on work supported by an RR\&D Research Career Development award (grant C3529V to Sherri L. Smith, PhD), an RR\&D Senior Research Career Scientist award (grant C2400S to Richard H. Wilson, PhD), and the RR\&D Auditory and Vestibular Dysfunction Research Enhancement Award Program (grant C4339F).

The authors have declared that no competing interests exist.

\section{REFERENCES}

1. Smith SL, Kricos PB, Holmes AE. Vision loss and counseling strategies for the elderly. Hear Rev. 2001;8(11):42, 44, 46, 56.

2. Brennan M, Horowitz A, Su YP. Dual sensory loss and its impact on everyday competence. Gerontologist. 2005;45(3): 337-46. [PMID: 15933274]

3. Keller BK, Morton JL, Thomas VS, Potter JF. The effect of visual and hearing impairments on functional status. J Am Geriatr Soc. 1999;47(11):1319-25. [PMID: 10573440]

4. Crews JE, Campbell VA. Vision impairment and hearing loss among community-dwelling older Americans: Implications for health and functioning. Am J Public Health. 2004; 94(5):823-29. [PMID: 15117707]

5. Reuben DB, Mui S, Damesyn M, Moore AA, Greendale GA. The prognostic value of sensory impairment in older persons. J Am Geriatr Soc. 1999;47(8):930-35. [PMID: 10443852]

6. Appollonio I, Frattola L, Carabellese C, Trabucchi M. The eyes and ears of the world of function. J Am Geriatr Soc. 1989;37(11):1099-1100. [PMID: 2809057]

7. Brennan M, Su YP, Horowitz A. Longitudinal associations between dual sensory impairment and everyday competence among older adults. J Rehabil Res Dev. 2006;43(6): 777-92. [PMID: 17310427]

8. Lupsakko T, Mantyjarvi M, Kautiainen H, Sulkava R. Combined hearing and visual impairment and depression in a population aged 75 years and older. Int J Geriatr Psychiatry. 2002;17(9):808-13. [PMID: 12221653]

9. Capella-McDonnall ME. The effects of single and dual sensory loss on symptoms of depression in the elderly. Int $\mathrm{J}$ Geriatr Psychiatry. 2005;20(9):855-61. [PMID: 16116571]
10. Chou KL, Chi I. Combined effect of vision and hearing impairment on depression in elderly Chinese. Int J Geriatr Psychiatry. 2004;19(9):825-32. [PMID: 15352139]

11. Chia EM, Mitchell P, Rochtchina E, Golding M, Foran S, Golding M, Wang JJ. Association between vision and hearing impairments and their combined effects on quality of life. Arch Ophthalmol. 2006;124(10):1465-70.

[PMID: 17030715]

12. Lam BL, Lee DJ, Gomez-Marin O, Zheng DD, Caban AJ. Concurrent visual and hearing impairment and risk of mortality: The National Health Interview Survey. Arch Ophthalmol. 2006;124(1):95-101. [PMID: 16401790]

13. Kart CS, Kinney JM. The realities of aging: An introduction to gerontology. 6th ed. Boston (MA): Allyn \& Bacon; 2001.

14. Dennis K. Dual sensory impairment: Challenges to rehabilitation. In: Proceedings of the Visual Impairment Services Team, Blind Rehabilitation Outpatient Specialist, and Audiology Training Conference; 2004 Aug 10-12; Reno, Nevada.

15. National Center for Rehabilitative Auditory Research; Department of Veterans Affairs Rehabilitation Research and Development. NCRAR Newsletter. Portland (OR): Department of Veterans Affairs Medical Center; 2004.

16. Cambell VA, Crews JE, Moriatry DG, Zack MM, Blackman DK. Surveillance for sensory impairment, activity limitation, and health-related quality of life among older adults-United States, 1993-1997. MMWR CDC Surveill Summ. 1999;48(8):131-56. [PMID: 10634273$]$

17. Lieberman D, Friger M, Lieberman D. Visual and hearing impairment in elderly patients hospitalized for rehabilitation following hip fracture. J Rehabil Res Dev. 2004;41(5): 669-74. [PMID: 15558396]

18. Fowler EP. Hearing standards for acceptance, disability rating, and discharge in the military and in industry. Laryngoscope. 1941;51(10):937-56.

19. Carhart R. Speech reception in relation to pattern of pure tone loss. J Speech Hear Dis. 1946;11:97-108.

20. Department of Veterans Affairs, Veterans Health Initiative. Hearing impairment. Washington (DC): Department of Veterans Affairs; 2002. Available from http://www1.va.gov/VHI

21. Harris JD. pure tone acuity and the intelligibility of everyday speech. J Acoust Soc Am. 1965;37:824-30. [PMID: 14285441]

22. Kryter KD, Williams C, Green DM. Auditory acuity and the perception of speech. J Acoust Soc Am. 1962;34(9A): 1217-23.

23. Wilson RH, McArdle R. Speech signals used to evaluate the functional status of the auditory system. J Rehabil Res Dev. 2005;42(4 Suppl 2):79-94. [PMID: 16470466]

24. World Health Organization [homepage on the Internet]. Available from World Health Organization: International 
Statistical Classification of Diseases and Health Related Problems. 10th Revision. Chapter VII, H54, Blindness and Low Vision. Available from: http://www.who.int/classifications/icd/en/

25. Department of Veterans Affairs; Veterans Health Initiative. Visual impairment and blindness. Washington (DC): Department of Veterans Affairs; 2002. Available from: http://www1.va.gov/VHI

26. Social Security Online [homepage on the Internet]. Baltimore (MD): Social Security Administration; c2008 [updated 2008 Jan 31; cited 2007 Jan 12]. If you are blind or have low vision-How we can help; [about 6 screens]. Available from: http://www.ssa.gov/pubs/10052.html

27. Congdon N, O’Colmain B, Klaver CC, Klein R, Munoz B, Friedman DS, Kempen J, Taylore HR, Mitchell P; Eye Diseases Prevalence Research Group. Causes and prevalence of visual impairment among adults in the United States. Arch Opthamol. 2004;122(4):477-85. [PMID: 15078664$]$

28. Vitale S, Cotch MF, Sperduto RD. Prevalence of visual impairment in the United States. JAMA. 2006;295(18): 2158-63. [PMID: 16684986]

29. Wang CC, Kosinski CJ, Schwartzberg JG, Shanklin AV. Physician's guide to assessing and counseling older drivers. Washington (DC): National Highway Traffic Safety Administration; 2003.

30. Goldstein DP. Hearing impairment, hearing aids and audiology. ASHA. 1984;26(9):24-35,38. [PMID: 6487402]

31. American National Standards Institute. Specification for audiometers. Melville (NY): Acoustical Society of America; 2004.

32. Carhart R, Jerger JF. Preferred method for clinical determination of pure tone thresholds. J Speech Hear Dis. 1959;24: 330-45.

33. Department of Veterans Affairs. Speech recognition and identification materials. Disc 4.0 (compact disc). Mountain Home (TN): Department of Veterans Affairs; 2004.

34. Agresti A, Finlay B. Statistical methods for the social sciences. 3rd ed. Upper Saddle River (NJ): Prentice Hall; 1997.
35. Owen DB. Handbook of statistical tables. Reading (MA): Addison-Wesley Publishing Company; 1962.

36. Green SB, Salkind NJ, Akey TM. Using SPSS for Windows: Analyzing and understanding data. 2nd ed. Upper Saddle River (NJ): Prentice Hall; 2000.

37. Cochran GC. Sampling techniques. 3rd ed. New York (NY): Wiley; 1977.

38. Mullins CJ, Bangs JL. Relationships between speech discrimination and other audiometric data. Acta Otolaryngol. 1957;47(2):149-57. [PMID: 13457874]

39. Gohdes DM, Balamurugan A, Larsen BA, Maylahn C. Age-related eye diseases: An emerging challenge for public health professionals. Prev Chronic Dis. 2005;2(3):A17. [PMID: 15963319]

40. Carhart R, Tillman TW. Interaction of competing speech signals with hearing losses. Arch Otolaryngol. 1970;91(3): 273-79. [PMID: 5414080]

41. Plomp R. Auditory handicap of hearing impairment and the limited benefit of hearing aids. J Acoust Soc Am. 1978; 63(2):533-49. [PMID: 670550]

42. Beattie RC. Word recognition functions for the CID W-22 in multitalker noise for normally hearing and hearingimpaired subjects. J Speech Hear Disord. 1989;54(1):20-32. [PMID: 2915523]

43. French NR, Steinberg JC. Factors governing the intelligibility of speech sounds. J Acoust Soc Am. 1945;17(1):103.

44. Smoorenburg GF. Speech reception in quiet and in noisy conditions by individuals with noise-induced hearing loss in relation to their tone audiogram. J Acoust Soc Am. 1992;91(1):421-37. [PMID: 1737889]

45. Fletcher DC, Schuchard RA. Visual function in patients with choroidal neovascularization resulting from agerelated macular degeneration: The importance of looking beyond visual acuity. Optom Vis Sci. 2006;83(3):178-89. [PMID: 16534460]

Submitted for publication February 1, 2007. Accepted in revised form August 29, 2007. 